\title{
A New Unsupervised Learning for Clustering Using Geometric Associative Memories`
}

\author{
Benjamín Cruz, Ricardo Barrón, and Humberto Sossa \\ Center for Computing Research - National Polytechnic Institute, \\ México City 07738, México \\ benji@helgrind.net, rbarron@cic.ipn.mx, hsossa@cic.ipn.mx \\ http://www.cic.ipn.mx
}

\begin{abstract}
Associative memories (AMs) have been extensively used during the last 40 years for pattern classification and pattern restoration. A new type of AMs have been developed recently, the so-called Geometric Associative Memories (GAMs), these make use of Conformal Geometric Algebra (CGA) operators and operations for their working. GAM's, at the beginning, were developed for supervised classification, getting good results. In this work an algorithm for unsupervised learning with GAMs will be introduced. This new idea is a variation of the k-means algorithm that takes into account the patterns of the a specific cluster and the patterns of another clusters to generate a separation surface. Numerical examples are presented to show the functioning of the new algorithm.
\end{abstract}

\section{Introduction}

Associative Memories (AMs) have been extensively used for many years in pattern recognition problems. An AM can be seen as an input-output system, see (11). When an input pattern $x$ is presented to an AM, it must to respond with the corresponding output pattern $y$.

$$
x \rightarrow M \rightarrow y
$$

The associative memories models developed until now can be categorized in three groups, those based on traditional vector algebra operations, those based on mathematical morphology operations, and those based on Geometric Algebra paradigm. The third group make use of Geometric Algebra [1] for their operations an operators, the so-called Geometric Associative Memories [2] (GAMs) are an example of memories that falls into this group.

The goal of GAMs is the classification of a pattern as belonging to a specific class if and only if the pattern is inside of the support region (hyper-sphere) of that class. Originally, GAMs were developed to function in a supervised form. In

\footnotetext{
* This work has been supported by the National Polytechnic Institute of Mexico (SIPIPN), under grants 20090620 and 20091421, by the Mexican Science and Technology National Council (CONACyT) under grants 46805 and 182938, and the European Comission and CONACyT under grant FONCICYT 93829.
}

E. Bayro-Corrochano and J.-O. Eklundh (Eds.): CIARP 2009, LNCS 5856, pp. 239-246, 2009.

(C) Springer-Verlag Berlin Heidelberg 2009 
this work, a new unsupervised learning algorithm for GAMs will be developed, it will be based on the well-known k-means [11] algorithm idea, but it will be use operators and operations of CGA for the building of the respective cluster.

\section{Basics on Geometric Algebra}

Geometric Algebras (GA's) also known as Clifford Algebras were introduced by William K. Clifford in 1878. He joined the works of Grassmann with the quaternion of Hamilton into a new mathematical model [1]. GA is a free coordinate geometric schema [5]. In GA, the geometric objects and the operators over these objects are treated in a single algebra 3. A special characteristic of GA is its geometric intuition. Another important feature is that, the expressions in GA usually have low symbolic complexity [7.

The Conformal Geometric Algebra (CGA) is a coordinate-free theory. In CGA, spheres and circles are both algebraic objects with a geometric meaning. In CGA, points, spheres, and planes are easily represented as multivectors. A multivector is the outer product of various vectors. CGA provides a great variety of basic geometric entities to compute with [7. In CGA the inner product is used for the computation of angles and distances.

For notations purposes, Euclidean vectors will be noted by lowercase italic letters $(p, q, s)$, with exception of the letters: $i, j, k, l, m, n$; these will be used to refer to indexes. The corresponding conformal points will be noted by uppercase italic letters $(C, P, S)$. A Euclidean matrix will be noted by a bold capital letter $(\mathbf{M}, \mathbf{H})$. To denote that an element belongs to an object (vector), a sub-script will be used. To refer that an object belongs to a set of objects of the same type, a superscript will be used. For example, let $S$ be a sphere, then $S_{k}$ is the $k$-th component of it, and $S^{k}$ is the $k$-th sphere of a set of spheres. To denote scalars Greek letters will be used $(\gamma, \varepsilon, \delta)$.

Let $p \in \mathbb{R}^{n}$ be an Euclidean point, it can be transformed to a CGA representation as:

$$
P=p+\frac{1}{2}(p)^{2} e_{\infty}+e_{0}
$$

where $e_{0}$ is the Euclidean origin and $e_{\infty}$ is the point at infinity such that $e_{0}^{2}=$ $e_{\infty}^{2}=0$ and $e_{0} \cdot e_{\infty}=-1$. Let $P$ and $Q$ two conformal points, the distance between them is found by means of the inner product [6] as follows:

$$
P \cdot Q=p \cdot q-\frac{1}{2}(p)^{2}-\frac{1}{2}(q)^{2}=-\frac{1}{2}(p-q)^{2} \Longleftrightarrow(p-q)^{2}=-2(P \cdot Q) .
$$

In the same way, a sphere takes the following representation [8]:

$$
S=C-\frac{1}{2}(\gamma)^{2} e_{\infty}=c+\frac{1}{2}\left((c)^{2}-(\gamma)^{2}\right) e_{\infty}+e_{0}
$$

where $C$ is the center point of the sphere in conformal notation as defined in (2), $\gamma$ is the radius of the sphere and $c$ is the Euclidean point of $C$. 
A distance measure between one conformal point $P$ and a sphere $S$ can be defined with the help of the inner product [10], as follows:

$$
P \cdot S=p \cdot s-\frac{1}{2}\left((s)^{2}-\frac{1}{2}(\gamma)^{2}\right)-\frac{1}{2}(p)^{2}=\frac{1}{2}\left((\gamma)^{2}-(s-p)^{2}\right)
$$

or in simplified form:

$$
2(P \cdot S)=(\gamma)^{2}-(s-p)^{2}
$$

Based on (6):

1. If $(P \cdot S>0)$ then $P$ is inside of $S$.

2. If $(P \cdot S<0)$ then $P$ is outside of $S$.

3. If $(P \cdot S=0)$ then $P$ is on $S$.

Therefore, in pattern classification if a CGA spherical neighborhood is used as support region, with the help of the inner product it is possible to know when a pattern is inside or outside of the region. In the same way, the distance between two points is easily computed with their inner product.

\section{Geometric Associative Memories for Clustering}

GAMs were developed, in principle, as a supervised classification model [2]. The training phase of that model is done by finding an optimal sphere with quadratic programming. In the classification phase an inner product between the unclassified pattern and the GAM must be applied. Then a minimum function is used to obtain an index class. GAMs can perfectly operate when the classes are spherically separable [2].

A GAM is precisely a matrix whose components are spheres, it can be seen in (7), where $m$ is the total number of classes. It uses spherical neighborhoods as decision regions.

$$
\mathbf{M}=\left[\begin{array}{c}
S^{1} \\
S^{2} \\
\vdots \\
S^{m}
\end{array}\right]
$$

Often, a clear distinction is made between learning problems than are supervised (classification) o unsupervised (clustering), the first one involving only labeled data while the latter involving only unlabeled data 4. However, clustering is a more difficult and challenging problem than classification [9]. The goal of data clustering is to find the natural grouping in a set of patterns, points, or objects without any knowledge of class label. In other words, it consists in to develop an automatic algorithm that will discover the natural grouping in the unlabeled data.

The K-means [11] is one of the simplest unsupervised learning algorithms that solve the clustering problem. The main idea is to define $k$ random centroids, one for each cluster. Then, to take each point of the data set and associate it to 
the nearest centroid using a distance measure. The new centroids must be recalculated as the centers of the clusters found. Then, with the new centers, the second step must be done, a loop has been generated. The loop is repeated until no more changes of centers are done.

The proposed algorithm is based on the previous ideas, but the Conformal Geometric Algebra paradigm will be used. Different to the k-means algorithm the clusters in this method will be found by separating the points of the associated centroid of the others points (the main advantage is that the new centroids are found automatically) solving an optimization problem, it is described in 22.

Given two sets of points $\left\{p^{i}\right\}_{i=1}^{l}$ and $\left\{p^{j}\right\}_{j=l+1}^{m}$, the idea is to find an optimal sphere $S$ with the least square error, such that $\left\{p^{i}\right\}$ are inside of $S$ and $\left\{p^{j}\right\}$ are outside of it. In other words, to solve:

$$
\min _{S}=\sum_{i=1}^{m}\left(P^{i} \cdot S\right),
$$

subject to (9) for points inside of the sphere and (10) for points outside of it.

$$
\begin{gathered}
P^{i} \cdot S \geq 0, i=1, \ldots, l . \\
P^{j} \cdot S<0, j=l+1, \ldots, l .
\end{gathered}
$$

That method takes into account the points inside and the points outside of the classification sphere for a better performance. Spheres function like attractors for the inside points and like contractors for the outside points, this gives a optimal separation surface. Another improvement is the computing of the distances among the centroids and the sets of points. This procedure is done with a GAM. The GAM is composed with the corresponding centroids. At the first iteration the centroids are randomly generated, at the following iterations the centroids are computing automatically when the corresponding convex hulls are built.

\subsection{New Clustering Algorithm}

Given a set of points $\left\{p^{i}\right\}_{i=1}^{m}$ in $\mathbb{R}^{n}$, and let $k$ the number of clusters:

1. Change $p^{i}$ to its conformal representation $P^{i}$, by using expr. (2), for $i=$ $1, \ldots, m$.

2. Set $k$ random centroids, $C^{j}$ where $j=1, \ldots, k$.

3. Set $\mathbf{M}=\left[C^{1}, \ldots, C^{k}\right]^{\prime}$

4. Generate $k$ clusters by associating every $P^{i}$ with the nearest centroids. It can be done with $\arg \min \left(M \cdot P^{i}\right)$ for $i=1, \ldots, m$.

5. Find $k$ spherical surfaces $S^{j}$ for each cluster by using eq. (8).

6. New values of $k$ centroids are the centers of each sphere.

7. Repeat step 3 until the values of $C^{j}$ do not change. 
As can be observed, in this algorithm the cluster computing is done by a single inner product and a minimum function, unlike the traditional k-means algorithm where a nearest neighbor algorithm to find the nearest centroid is used. Regarding computational complexity of both algorithms, it is known that the k-means algorithm can be solved in time $\Theta\left(m^{n k+1} \log m\right)$ [12, where $m$ is the number of points to be clustered, $n$ is the dimension of that points, and $k$ is the number of clusters.

In the case of the proposal, most of the time is spent on generating cluster associations among points and clusters (step 4). One such operation costs $\Theta(k)$. The new clusters generation $\operatorname{costs} \Theta(k m n)$, so its overall complexity is $\Theta\left(k^{2} m n\right)$. For a fixed number of iterations $I$, the overall complexity is therefore $\Theta\left(I k^{2} m n^{2}\right)$. Thus, our proposal has a linear complexity into the Conformal Geometric Algebra framework.

Another improvement of our algorithm is that at the end, in some situations, some points can be outside of all the spheres which can be both and advantage or disadvantage, depending on the characteristics of the problem.

Sometimes, that points can be noise. And they can be omitted without lossing essential information about the nature of the problem. But, they can be essential for most clustering problems.

\section{A Representative Example}

Due space limitations, one numerical example will be shown. Let the set of points in $\mathbb{R}^{2}$ shown in Figure 1 to be clustered. In this image, as it can be observed, four cumulus of points are visible at first appearance.

When the algorithm of the section 3.1 is applied, the graphs of the Figures 2. 3. and 4 are obtained. The solution in each case, as in most clustering problems, depends, mainly, of the first random centroids. Best solution is shown in Figure 4]

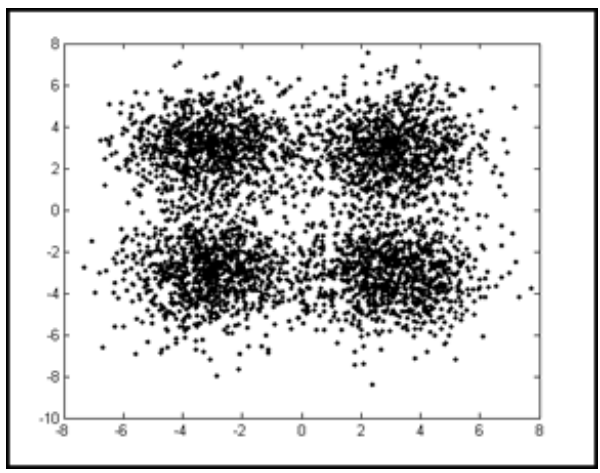

Fig. 1. Set of points in $\mathbb{R}^{2}$ to be clustered 


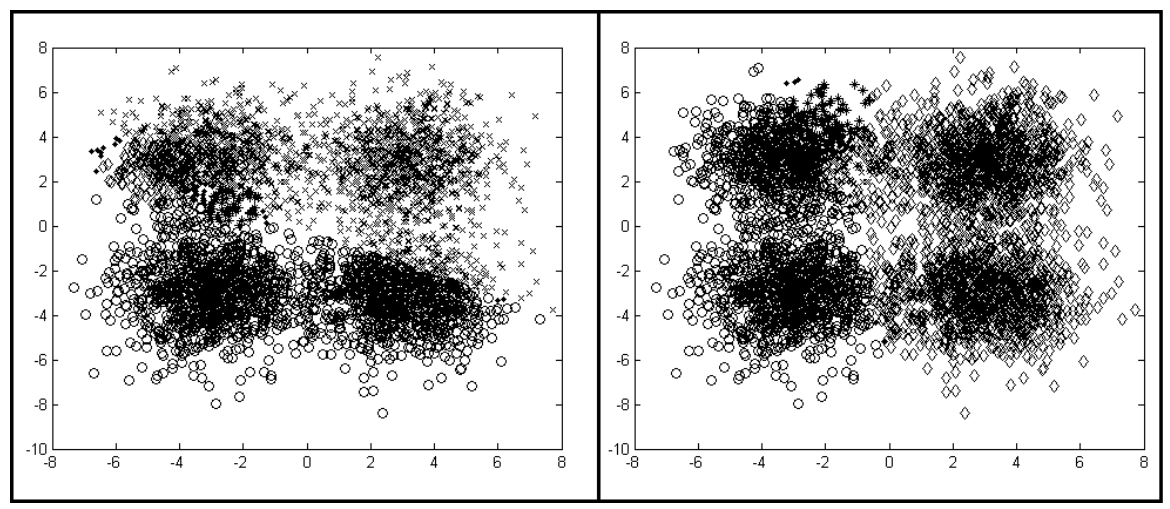

Fig. 2. Different solutions that solve the clustering problem of Fig. 1

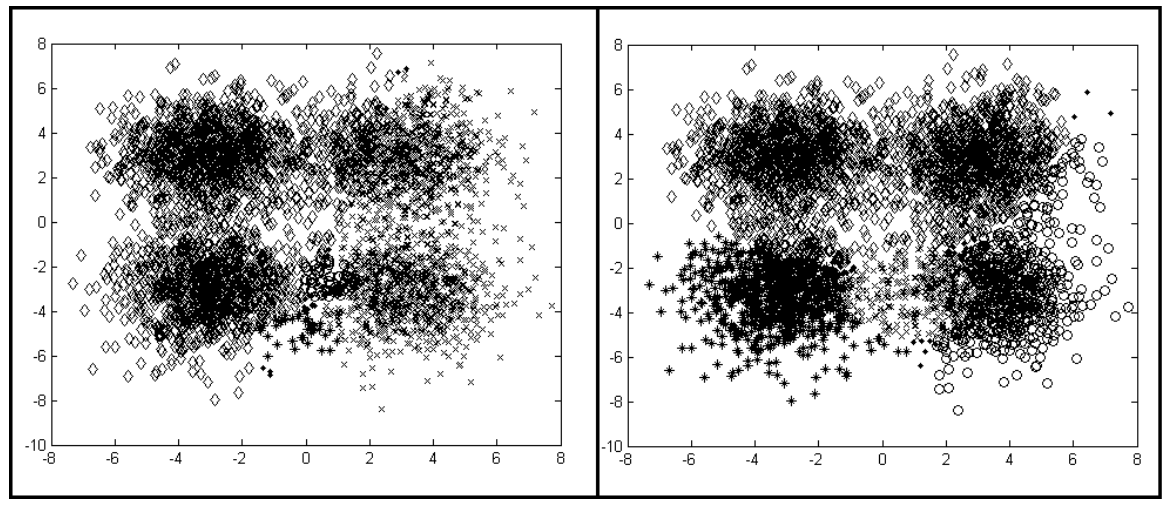

Fig. 3. Another solutions that solve the clustering problem of Fig. 1

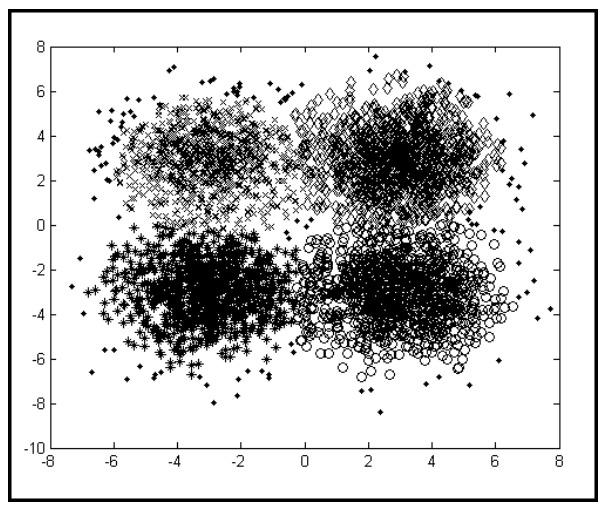

Fig. 4. Better solution that solves the clustering problem of Fig. 1 
In most of the graphs presented, some black points appeared, these points are outside of all the spheres, furthermore these pointds do not belong to a specific class. This is due the type of separation surface that is being used. A better approximation can be obtained with the use of other irregular shapes unlike the spheres or squares. But, depending on the type of problem this could be an advantage

\section{Conclusions and Ongoing Work}

An Associative Memory (AM) is an input-output device used in pattern recognition and pattern classification. Many AM models make use of traditional algebra or mathematical morphology for their working. Essentially, AMs were developed in a principle for supervised classification or pattern recognition. Recently a new kind of AM was developed, the so-called Geometric Associative Memory (GAM) are based on Conformal Geometric Algebra (CGA) paradigm for their operations and operators.

GAMs have been developed for supervised pattern classification getting good results. In this work a new algorithm for GAMs was introduced, this new algorithm can solve the clustering problem, it is based on the well-known k-means algorithm, but it works into the CGA framework.

New clusters are generated using an inner product between the GAM itself and one unclassified point, unlike the traditional k-means algorithm where all the distances among the current centroids and each point must be applied. It reduces the complexity of the algorithm.

Unlike traditional k-means algorithm. In the proposed algorithm some initial points can not be considered as belonging to a specific class. In some type of problems this can be an advantage because these points could be spurious points or noise. One numerical example was presented to test the algorithm. Most of the cases, best solution depends, mainly, of the first random centroids.

Nowadays, we are also interested to test our method in more realistic situations and in comparison (in computing time and performance) between the proposed model and other clustering models. We are working too in GAMs that work with separation surfaces other than spheres; like ellipses, squares or another irregular shape.

\section{References}

1. Clifford, W.: Applications of Grassmann's Extensive Algebra. Am. J. of Math. 1(4), 350-358 (1878)

2. Cruz, B., Barrón, R., Sossa, H.: Geometric Associative Memories and their Applications to Pattern Classification. In: Bayro-Corrochano, E., Sheuermann, G. (eds.) Geometric Algebra Computing for Computing Science and Engineering. Springer, London (2009) (to be published)

3. Dorst, L., Fontijne, D.: 3D Euclidean Geometry Through Conformal Geometric Algebra (a GAViewer Tutorial). University of Amsterdam Geometric Algebra Website (2005), http://www.science.uva.nl/ga/tutorials/CGA/ 
4. Duda, R., HArt, P., Stork, D.: Pattern Classification. John Wiley \& Sons, New York (2001)

5. Hestenes, D., Sobczyk, G.: Clifford Algebra to Geometric Calculus. Kluwer/Springer (1984)

6. Hestenes, D., Li, H., Rockwood, A.: New Algebraic Tools for Classical Geometry. In: Sommer, G. (ed.) Geometric Computing with Clifford Algebras, vol. 40, pp. 3-23. Springer, Heildeberg (2001)

7. Hildebrand, D.: Geometric Computing in Computer Graphics Using Conformal Geometric Algebra. Tutorial, TU Darmstadt, Germany. Interact. Graph. Syst. Group (2005)

8. Hitzer, E.: Euclidean Geometric Objects in the Clifford Geometric Algebra of Origin, 3-Space. Infinity. Bull. of the Belgian Math. Soc. 11(5), 653-662 (2004)

9. Jain, A.: Data Clustering: 50 Years Beyond K-means. In: Daelemans, W., Goethals, B., Morik, K. (eds.) ECML PKDD 2008, Part I. LNCS (LNAI), vol. 5211, pp. 3-4. Springer, Heidelberg (2008)

10. Li, H., Hestenes, D., Rockwood, A.: Generalized Homogeneous Coordinates for Computational Geometry. In: Sommer, G. (ed.) Geometric Computing with Clifford Algebras, vol. 40, pp. 27-52. Springer, Heildeberg (2001)

11. MacQueen, J.: Some Methods for Classification and Analysis of Multivariate Observations. In: Proc. of 5-th Berkeley Symp. on Math. Stats. and Prob., vol. 1, pp. 281-297. Unversity of California Press, Berkeley (1967)

12. Inaba, M., Katoh, N., Imai, H.: Applications of weighted Voronoi diagrams and randomization to variance-based k-clustering. In: Proceedings of 10th ACM Symposium on Computational Geometry, pp. 332-339 (2004) 